\title{
Primary Intracranial Malignant Melanomas: Retrospective Analysis of Management in a Single Chinese Institution and literature Review
}

\section{Lifeng Chen}

The first medical center of the Chinese PLA General Hospital

\section{Yang Yang}

The second medical center of the Chinese PLA General Hospital

Dongmei Li

The second medical center of the Chinese PLA General Hospital

\section{$\mathrm{Bo} \mathrm{Bu}$}

The first medical center of the Chinese PLA General Hospital

xiaodong ma ( $\nabla$ maxiaodong301@126.com )

The first medical center of the Chinese PLA General Hospital https://orcid.org/0000-0002-5404-6480

\section{Research}

Keywords: Malignant melanoma, Central nervous system, Diagnosis, Treatment

Posted Date: September 30th, 2021

DOI: https://doi.org/10.21203/rs.3.rs-927214/v1

License: (c) (i) This work is licensed under a Creative Commons Attribution 4.0 International License. Read Full License 


\section{Abstract}

Background: Primary intracranial malignant melanoma (PIMM) is a rare malignant tumor. The authors retrospectively reviewed and discussed the clinical features, treatment modalities, and clinical outcomes of patients with histologically proven PIMM.

Methods: The data of 15 patients with PIMM in our hospital within 14 years (from January 2005 to January 2019) were collected. The clinical and imaging presentations, pathology, surgical strategies, adjuvant treatment and the prognosis were analyzed in this study.

Results: Eleven men and 4 women with mean age 37.9 years (19-61 years) were observed over an average follow-up period of 22.6 months (range, 6-36 months). CT showed iso or high density in 12 cases (80\%). MRI sacns indicated that 14 tumors were mainly hyperintensity on T1 weighted images, hypointensity on T2 weighted images, and had no or mild enhancement. The treatment modalities included total resection followed by conventional radiotherapy (RT) $(n=12)$, and subtotal resection followed by stereotactic radiosurgery (SRS) $(n=3)$. Fifteen cases had recurrence or metastasis at the average 14.7 months (6-23 months): local recurrence ( 8 cases), distant metastasis ( 5 cases), both of them ( 2 cases). Fourteen cases ( $93.3 \%$ ) died and the mean overall survival was 22 months (6-36 months). The median survival period was 23 months. The overall survival rates at 1, 2 and 3 years were $80 \%, 47 \%$, and $13 \%$, respectively. Radical resection with RT was associated with longer overall survival (log-rank, $p<0.05$ ).

Conclusions: PIMM is an extremely rare tumor with poor prognosis, which is difficult to get correct preoperative diagnosis. Improvement of the recognition of MRI features of melanoma can increase the preoperative diagnosis rate, and radical resection with RT may provide longer overall survival rate. Targeted and immunotherapy therapies may provide promise as treatment options for PIMM.

\section{Background}

Primary intracranial malignant melanoma (PIMM) is a rare tumor, representing $0.07 \%$ of all intracranial tumors ${ }^{(1)}$. It accounts for about $1 \%$ of all melanoma cases ${ }^{(2)}$. Because of the rarity of PIMMs and the lack of randomized study, there are the high chance of misdiagnosis and limited knowledge regarding therapy. Resection or biopsy combination with adjuvant treatment, including whole-brain radiotherapy (WBRT), stereotactic radiosurgery (SRS), chemotherapy, and/or immunotherapy are recommended ${ }^{(3)}$. Gross total resection can improve the outcomes of PIMMs ${ }^{(3)}$. But the benefit of radiation therapy, chemotherapy and immunotherapy is still controversy, and the prognosis of the disease is poor. In the present study, we retrospectively reviewed the therapeutic strategies and follow-up combined with literature reviewing to better understand the clinical characteristics and the therapeutic schedule.

\section{Material And Methods}




\section{Patients}

During a 14-year period from January 2005 to January 2019, there were 15 consecutive patients with pathologically confirmed PIMM were treated in the Department of Neurosurgery of our hospital including 11 men and 4 women with mean age 37.9 years (19-61 years).

The study was approved by the Ethics Board of the Chinese People's Liberation Army (PLA) General Hospital. Patients with a postoperative pathological diagnosis of malignant melanoma were included based on the following criteria outlined by Willis ${ }^{(1,4)}$ : no skin or eye melanoma ${ }^{(2)}$; no history of skin or eyes melanoma surgery ${ }^{(3)}$; no extracranial viscera melanoma metastases. The clinical information including the clinical presentation, imaging, treatment, and follow-up were retrospectively collected and analyzed from medical records, clinical notes and surgical reports.

\section{Patient Evaluation}

CT imaging was performed in 12 patients. Magnetic resonance imaging (MRI) was performed for all 15 patients before and after surgical treatment. Tumor size was defined as the greatest tumor diameter on MRI. Gross total resection (GTR) was defined as $100 \%$ gross resection of the tumor. There was no residual tumor on MRI within 3 months after operation. Subtotal resection (STR) is close to total resection, but the purpose is incomplete resection. The postoperative MRI showed tumors that increased in size were considered as tumors recurrence or regrowth. All 15 patients were followed up by neuroimaging and neurological examination. Neurological and imaging examinations were carried out preoperatively, at 3 months postoperatively, and at 1 year to 3 years intervals after surgery. The Karnofsky Performance Status (KPS) score was also determined for each patient based on clinical evaluation.

Surgical tumor specimens were fixed in formaldehyde and embedded in parain. It underwent microscopic and immunohistology-chemical analysis. Two senior neuropathologists independently verified the histological examinations.

\section{Statistical Methods}

The characteristics of patients were described in descriptive statics. Kaplan-Meier method was used to analyze overall survival (OS); P-values are considered significant if less than 0.05 . All statistical analyses were performed using SPSS, version 19.0 (SPSS, Chicago, IL, USA).

\section{Results}

\section{Preoperative characteristics}


The duration of preoperative symptoms was 6 days to 2 years, with an average of 6.3 months. The demographics and presenting symptoms of 15 patients are described in detail in Table 1. Twelve CT scans and $15 \mathrm{MRI}$ scans were performed. CT showed iso or high density in 12 cases (80\%) (Figure1a, Figure2a, Figure3a, Figure4a). MRI showed short T1 and slightly short T2 in 14 cases (93.3\%) (Figure 1b, $c$, Figure $2 b, c$, Figure $3 c, d$, Figure $4 b, c)$. The tumors showed mild or no enhancement on enhanced MRI (Figure $3 \mathrm{e}$ ). The maximum diameter of tumor was $2.5-8.0 \mathrm{~cm}$, with an average of $4.6 \mathrm{~cm}$. 14 patients had solitary tumors (93.3\%). One patient had two tumors located in the supratentorial region. Peritumoral brain edema was observed in 3 cases (20\%). Based on the preoperative MRI, three patients (20\%) were accurately diagnosed with melanoma. Other patients were misdiagnosed as glioma, metastasis, schwannomas or meningiomas.

\section{Table 1}

Summary of 15 patient's characteristics with primary malignant intracranial melanomas. 


\begin{tabular}{|ll|}
\hline Characteristics & $\mathrm{n}=15$ \\
\hline Demographics & \\
\hline Mean age, years & 37.9 (range $19-61)$ \\
\hline Males: Females & $11: 4$ \\
\hline Major symptoms and signs & \\
\hline Headache & $10(67 \%)$ \\
\hline Vomit & $2(13 \%)$ \\
\hline Weakness of left limb & $1(7 \%)$ \\
\hline Nystagmus & $1(7 \%)$ \\
\hline Epilepsy & $1(7 \%)$ \\
\hline Tinnitus and deafness & $1(7 \%)$ \\
\hline Numbness of left face & $1(7 \%)$ \\
\hline Facial paralysis & $1(7 \%)$ \\
\hline Mean symptom duration, months & $6.3($ range 6 days-2 years) \\
\hline Tumor location & \\
\hline Cerebral & $9(60 \%)$ \\
\hline CPA & $3(20 \%)$ \\
\hline Cerebellum & $1(7 \%)$ \\
\hline Foramen magnum & $1(7 \%)$ \\
\hline Middle fossa & $1(7 \%)$ \\
\hline Mean size, cm & $4.6($ range $2.5-8.0)$ \\
\hline Median pre-operative KPS & $80($ range70-90) \\
\hline
\end{tabular}

\section{Surgical characteristics}

The tumors were removed through the different approaches according to the location of tumor. During the operation, the dura mater or the surrounding brain tissue were found to be black in 6 cases, the tumor was black (Figure1e, Figures 2e, f) in 14 cases and tan appearance in 1 case. Tumors were soft and mild or moderate vascularized. Two cases of supratentorial tumor had hemorrhage. Intraoperatively, the involved the section of the dura involved with the tumors was coagulated or removed. GTR was achieved 
in 12 cases (80\%) (Figures 1d, g, Figure 2d, Figure 3f, Figure 4d). STR was achieved in 3 cases $(20 \%)$ because of their close relationship with pyramidal tract.

There was no perioperative mortality. Facial paralysis was observed in one patient. He recovered after three months. One patient had postoperative intracranial infection. He was successfully treated with continuous drainage of cerebrospinal fluid for 5 days. At time of discharge, the preoperative neurological function was maintained or improved in 14 patients (93.3\%). The KPS scores ranged from 70 to 100 (Table 2).

Table 2

Outcome of treatment in 15 patients with primary malignant intracranial melanomas.

\begin{tabular}{ll}
\hline Outcome & Total \\
Grade of resection & \\
\hline Total resection & 12 \\
\hline Subtotal resection & 3 \\
\hline Recurrence after first treatment & \\
\hline local recurrence alone & $8(53 \%)$ \\
\hline Metastasis alone & $5(33 \%)$ \\
\hline Both & $2(13 \%)$ \\
\hline Location of metastasis & \\
\hline Cerebellar & $2(40 \%)$ \\
\hline Occipital lobe & $2(40 \%)$ \\
\hline Multiple cerebral metastases & $1(20 \%)$ \\
\hline Time to local recurrence( $\mathrm{n}=10)$ Mean, months & $13.6($ range, $6-20)$ \\
\hline $\begin{array}{l}\text { Time to metastasis }(\mathrm{n}=7) \text { Mean, months } \\
\text { toextraneuralmetastasis(n=5)Mean,months }\end{array}$ & $16.5($ range, $12-$ \\
\hline Time to overall death(n=14) & $23)$ \\
\hline Mean, months & \\
\hline Mean follow-up duration, months & $22($ range, 6-36) \\
\hline Median post-operative KPS & $22.6($ range, 6-36) \\
\hline
\end{tabular}

\section{Postoperative course and follow-up}


Adjuvant treatments included conventional radiotherapy (RT) and stereotactic radiosurgery (SRS). No patients received adjuvant chemotherapy. All 12 patients with GTR underwent postoperative conventional RT with a mean dose of $50 \mathrm{~Gy}$ (range, 45-54 Gy) in 1.8-2 Gy fractions. Three patients with STR received Cyber Knife radiosurgery (CKRS; Accuray, Sunnydale, CA, USA). The average follow-up was 22.6 months (range, 6-36 months). All 15 patients were independent at 1 month postoperatively. Fifteen cases had recurrence or metastasis (Figure $1 \mathrm{~h}$ ) at the average 14.7 months (6-23 months): local recurrence (8 cases), distant metastasis ( 5 cases), both of them ( 2 cases). Of the 15 patients, 4 patients received second surgery, 1 patient received radiosurgery. The average progression free survival time was 16 and 8 months in the GTR combined with RT and STR combined with CKRS groups, respectively (a difference of 8 months, $p=0.001$; Figure 5). The mean overall survival was 22 months (6-36 months) (Figure 6). The median survival period was 23 months (Table 1). Fourteen patients died due to the tumor. The overall survival rates at 1,2 and 3 years were $80 \%, 47 \%$, and $13 \%$, respectively. The average survival time was 25 and 9 months in the GTR combined with RT and STR combined with CKRS groups, respectively (a difference of 16 months, $p=0.000$; Figure 7 ).

\section{Pathological examination}

The senior neuropathologist verified the histological examinations. Microscopic examination showed the typical histological diagnosis of malignant melanoma (Figure 1f, Figure $2 \mathrm{~g}$ ). The most tumor cells contained rich melanin and large nuclei. The giant polygonal or spindle-shaped cells could be seen, and mitosis was usually noted. Immunohistochemical analysis demonstrated the positive rates of S-100 protein, antimelanoma antibody (HMB-45) and Vimentin (VIM) were 14 cases (93.3\%), 13 cases (86.7\%) and 13 cases $(86.7 \%)$ respectively. The marker of proliferation $\mathrm{Ki}-67$ labeling index in all the tumors ranged from $10 \%$ to $30 \%$.

\section{Discussion}

\section{Epidemiology and clinical features}

Primary intracranial melanoma is a rare tumor in the central nervous system (CNS), which was first described by Virchow in $1859^{(5)}$. The estimated incidence is 0.5 cases per 10, 000, 000 person-years in the literature ${ }^{(6)}$. Malignant intracranial melanomas can be divided into two types including primary and secondary subtypes. The diagnosis of PIMMs should be consistent with that outlined by Willis ${ }^{(4)}$. In this report, perioperative examination demonstrated all patients had no melanoma or non-brain melanoma surgical history in the other parts of body, and all of 15 patients were diagnosed with PIMM. While the reports of sex predominance in literature are inconsistent, and some studies showed male predominance $(5,6)$. There was male predominance (male: female $=11: 4$ ) in our series, which is consistent with the literature ${ }^{(7)}$. The mean age of onset was 37.9 years (19-61 years), which is younger than that in the literature ${ }^{(1)}$. 
PIMMs were divided into diffuse meningeal tumors and solitary melanomas by Gibson in 1957 (3). Although PIMMs can occur throughout the central nervous system, they are more likely to develop in the posterior cranial fossa, Meckel cave, and spinal cord, and often present with mass effect ${ }^{(5)}$. In this study, 5 cases located Infratentorial, and 1 case located in middle and posterior cranial fossa. The clinical symptoms of PIMM are nonspecific and consistent with those of other malignant brain tumors. The most common clinical symptoms were intracranial hypertension and hydrocephalus (43.2\%) in the literature ${ }^{(5)}$, and the diffuse type has a higher possibility of intracranial hypertension and hydrocephalus than the solitary type ${ }^{(3)}$. While the 14 cases were the solitary type tumors in this report. Symptoms at presentation include headache, vomiting, and focal neurological deficits. The most common clinical symptoms were headache, vomiting due to intracranial hypertension. The mean duration of symptoms was 6.3 months, longer than common intracranial malignant tumors.

\section{Radiology}

CT and MRI are very important for preoperative differential diagnosis of PIMMs. Typical PIMMs may show high density in CT scan, which needs to be differentiated from hemorrhage (Figure1a, Figure2a, Figure3a, Figure4a). But some PIMMs also display equal or low density in CT scan, and generally lack specificity. MRI is still the gold imaging diagnosis standard of PIMMs. MRI scans can reveal typical features of most PIMMs, which are high signal on T1 weighted images and low signal on T2 weighted images. It is different from that of other common intracranial tumors including meningioma, schwannoma, metastasis, and glioma. There is a bipolar dipole interaction between the unpaired electrons of the stable melanin organic radicals and the aquaporin, resulting in the shortening of T1 and T2 relaxation time and the generation of typical MRI features. The decrease of relaxation time is directly proportional to the content of melanin in melanoma ${ }^{(8)}$.

Most of tumors with hyperintensity on T1 weighted images indicate hemorrhage, fat, or melanin. Furthermore, hypointensity on T2 weighted images may be a clue to distinguish lipoma from melanoma. Typical T1 and T2 weighted signals may provide clues for diagnosis of PIMMs. In this study, 14 tumors were mainly hyperintensity on T1 weighted images, hypointensity on T2 weighted images, and had no or mild enhancement. But only three patients were accurately diagnosed with melanoma before surgery. The definite diagnosis was not made until a typical black tumor was found during the operation. Intratumor hemorrhage which leads to MRI signal confusion and the rarity of melanoma make the correct initial diagnosis very difficult. However, the enhancement of the recognition to MRI features of melanoma can improve the preoperative diagnosis rate.

\section{Histopathology and differential diagnosis}

Pathological examination is standard protocol for diagnosis. Malignant melanomas can be diagnosed by routine $\mathrm{H} \& \mathrm{E}$ and immunohistochemically staining techniques. The cells of malignant melanomas are 
densely pleomorphic, spindle -shaped cells with mitosis and abundant cytoplasm with melanin deposits ${ }^{(4)}$. In this report, the tumor tissue rich in melanin (Figure 1f), heteromorphous large cells with obvious nucleoli (Figure $2 \mathrm{~g}$ ) and giant tumor cells can be seen under a light microscope.

Immunohistochemically staining can differentiate malignant melanomas from other tumors. S-100 is highly sensitive (95\%) to malignant melanomas, and HMB-45 is another highly sensitive and specific pathological marker for diagnosing malignant melanomas ${ }^{(5,9)}$. Vimentin $(\mathrm{VIM})$ is a mesenchymal tumor marker, which can provide a complementary effect in the diagnosis and the differential diagnosis of PIMMs combined with other markers ${ }^{(5,9)}$. In this report, the positive rates of S-100, HMB-45 and vimentin were $93.3 \% \%, 86.7 \%$ and $86.7 \%$, respectively, which were consistent with the literature.

\section{Treatments and outcomes}

Most authors $(1,5,7,10,11)$ agree gross total resection is the most important treatment for melanoma of the central nervous system. The incomplete removal increases the risk of recurrence and poor prognoses. The prognoses of the patients that received total resection are better than those of the patients who received incomplete resection $(3,11)$. Total resection combined with postoperative radiotherapy seems to be the preferred treatment for eliminating mass effect, improving preoperative symptoms, and achieving a histological diagnosis ${ }^{(3)}$. Rodriguez ${ }^{(7)}$ reported the mean survival in patients who received total removal of their tumor (19.6 months) was significantly longer than that in patients who underwent partial resection or biopsy ( 9.3 months). Man ${ }^{(6)}$ reported complete surgical resection could increase the survival rate, while the age of less than 19 years and intracranial tumor were independent factors of poor prognosis. Subtotal resection or biopsy plus radiotherapy (chemotherapy) cannot change the survival of

patients ${ }^{(5,9,12)}$. Our current study further validates the results. GTR with RT appeared to extend progression free survival. The average overall survival time of the GTR group was significantly higher than that in the STR group ( 25 versus 9 months, $p=0.000$ ). Surgical resection is based on the patients' symptoms, the location, size and number of the lesion. Total resection should be attempted with microsurgical techniques on the basis of protecting nerve function. But it is difficult to achieve total resection because of the occult onset of the tumor, the abundant blood supply of the tumor and the close relationship between the tumor and the important neurological structure. In this group, GTR was achieved in $80 \%$ cases, and STR was achieved in $20 \%$ cases because the tumors had very close relationship with pyramidal tract.

Yamane ${ }^{(13)}$ reported the mean survival in patients with solitary tumors was 20.7 months. Man ${ }^{(6)}$ reported that the 1-year, 2-year, 3-year and 5-year survival rates of primary CNS melanoma were 89.3\%, $75.6 \%, 65.2 \%, 37.7 \%$ respectively, and the median survival rate was 15 months. The median survival time was 23 months in this group. The treatment effect is basically the same as that reported in the literature, but due to the small number of cases, it is necessary to further increase the number of cases to analyze the treatment results. 
Although many scholars reported that melanoma is not sensitive to the commonly used doses radiotherapy, some studies also showed that the addition of adjuvant radiotherapy to surgery

significantly reduces the local recurrence risk compared with resection alone ${ }^{(11,14-17)}$. The combined application of WBRT and SRS is more effective than that of WBRT and SRS alone ${ }^{(11,15)}$. The prognosis of patients undergoing microsurgery combined with SRS and / or WBRT is better than that of patients undergoing microsurgery or WBRT ${ }^{(11,15)}$. In this study, 12 patients with GTR received adjuvant RT postoperatively, and other 3 patients with STR received CKRS. The average overall survival time of the GTR combined with RT group was significantly higher than that of STR combined with CKRS group.

The role of adjuvant chemotherapy for PIMMs is controversial. There is little evidence that chemotherapy carries significant effect for PIMMs. Chemotherapy drugs have not efficacy because they cannot penetrate the blood-brain barrier ${ }^{(11)}$. some authors reported when the tumors grow within the brain parenchyma, the blood-brain barrier is damaged in structure and function, increasing the permeability ${ }^{(11)}$. Adjuvant chemotherapy has shown limited effect in the management of metastatic melanoma ${ }^{(11)}$. There was no patient received postoperative chemotherapy in our series. Some authors ${ }^{(11,18-22)}$ reported that immunotherapy could prolong the overall survival of metastatic melanoma patients. Some clinical investigators ${ }^{(11,23,24)}$ were also exploring the effect of gene therapy (targeted therapy) on PIMMs. Gene therapy (targeted therapy) combined with immunotherapy could improve the prognosis of metastatic melanoma better than immunotherapy combined with radiotherapy ${ }^{(25)}$. These results also need further evaluation.

\section{Limitation}

A Solitary tumor with leptomeningeal enhancement could be have in a manner similar to diffuse leptomeningeal melanosis, which is considered be a benign feature, but with a very poor prognosis and a mean survival of 6.7 months ${ }^{(1)}$. In this series we excluded the patients with diffuse leptomeningeal enhancement who had no surgical indication. More patients and studies are required to confirm the role and efficacy of surgery and adjuvant therapy for PIMM. The incidence rate of this tumor is low, most of which are reported in the form of case report in literature. It is difficult to compare our report with other series in the literature.

\section{Conclusions}

PIMM is an extremely rare tumor with poor prognosis, which is difficult to get correct preoperative diagnosis. Improvement of the recognition of MRI features of melanoma can increase the preoperative diagnosis rate, and radical resection with RT may provide longer overall survival rate. Targeted and immunotherapy therapies may provide promise as treatment options for PIMM.

\section{Abbreviations}


CKRS:Cyber Knife radiosurgery;CNS: Central nervous system;CPA: Cerebellopontine angle;GTR:Gross total resection;MRI:Magnetic resonance imaging;PIMM:Primary intracranial malignant melanoma;RT: radiotherapy;SRS: stereotactic radiosurgery;STR: Subtotal resection;VIM:Vimentin;WBRT:whole-brain radiotherapy.

\section{Declarations}

\section{Acknowledgements}

Not applicable.

\section{Authors' contributions}

LC, YY, and XM contributed to the conception, design, and data analysis and drafted and revised the manuscript. DL and YY collected and analyzed the data. BB reviewed the manuscript. LC drew the simulated diagram of the manuscript. All authors read and approved the final manuscript.

\section{Funding}

No funding.

\section{Availability of data and materials}

The datasets used and/or analyzed during the current study are available from the corresponding author on reasonable request.

\section{Ethics approval and consent to participate}

The studies involving human participants were reviewed and approved by Ethics Committee of the Chinese People's Liberation Army (PLA) General Hospital.

\section{Consent for publication}

The patients/participants provided their written informed consent to participate in this study. Written informed consent was obtained from the individual(s) for the publication of any potentially identifiable images or data included in this article. 


\section{Competing interests}

The authors declare that the article content was composed in the absence of any commercial or financial relationships that could be construed as a potential conflict of interest.

\section{Author details}

${ }^{1}$ Department of Neurosurgery, The first medical center of the Chinese PLA General Hospital, No.28 Road Fuxing, Haidian District, Beijing, China.

${ }^{2}$ Department of Neurology, The second medical center of the Chinese PLA General Hospital, No.28 Road Fuxing, Haidian District, Beijing, China.

\section{References}

1. Arai N, Kagami H, Mine Y, Ishii T, Inaba M. Primary Solitary Intracranial Malignant Melanoma: A Systematic Review of Literature. World Neurosurg (2018) 117:386 - 93. Epub 2018/07/01. doi: 10.1016/j.wneu.2018.06.138. PubMed PMID: 29959081.

2. Balakrishnan R, Porag R, Asif DS, Satter AM, Taufiq M, Gaddam SS. Primary Intracranial Melanoma with Early Leptomeningeal Spread: A Case Report and Treatment Options Available. Case Rep Oncol Med (2015) 2015:293802. Epub 2015/08/22. doi: 10.1155/2015/293802. PubMed PMID: 26294993; PubMed Central PMCID: PMCPMC4532938.

3. Li CB, Song LR, Li D, Weng JC, Zhang LW, Zhang JT, et al. Primary intracranial malignant melanoma: proposed treatment protocol and overall survival in a single-institution series of 15 cases combined with 100 cases from the literature. J Neurosurg. 2019;132(3):902-13. doi: 10.3171/2018.11.JNS181872. PubMed PMID: 30835686.

4. Wang J, Guo ZZ, Wang YJ, Zhang SG, Xing DG. Microsurgery for the treatment of primary malignant intracranial melanoma: a surgical series and literature review. Eur J Surg Oncol. 2014;40(9):106271. doi: 10.1016/j.ejso.2013.11.024. PubMed PMID: 24360613.

5. Liubinas SV, Maartens N, Drummond KJ. Primary melanocytic neoplasms of the central nervous system. J Clin Neurosci. 2010;17(10):1227-32. doi:10.1016/j.jocn.2010.01.017. PubMed PMID: 20558070. Epub 2010/06/19.

6. Man W, Wang G. Incidence. Outcomes and Predictors of Primary Central Nervous System Melanoma: A SEER-Based Study. World Neurosurg. 2019;129:e782-e90. doi:10.1016/j.wneu.2019.06.030. PubMed PMID: 31203063. Epub 2019/06/17.

7. Rodriguez y Baena R, Gaetani P, Danova M, Bosi F, Zappoli F. Primary solitary intracranial melanoma: case report and review of the literature. Surg Neurol. 1992;38(1):26-37. doi:10.1016/0090- 
3019(92)90208-5. PubMed PMID: 1615371. Epub 1992/07/01.

8. Barkovich AJ, Frieden IJ, Williams ML. MR of neurocutaneous melanosis. AJNR Am J Neuroradiol. 1994;15(5):859-67. Epub 1994/05/01. PubMed PMID: 8059652; PubMed Central PMCID: PMCPMC8332186.

9. Sloan AE, Nock CJ, Einstein DB. Diagnosis and treatment of melanoma brain metastasis: a literature review. Cancer Control. 2009;16(3):248-55. Epub 2009/06/27. doi: 10.1177/107327480901600307. PubMed PMID: 19556965.

10. Suranagi VV, Maste P, Malur PR. Primary intracranial malignant melanoma: A rare casewith review of literature. Asian J Neurosurg. 2015;10(1):39-41. doi:10.4103/1793-5482.151508. PubMed PMID: 25767575; PubMed Central PMCID: PMCPMC4352627. Epub 2015/03/15.

11. Gibney GT, Forsyth PA, Sondak VK. Melanoma in the brain: biology and therapeutic options. Melanoma Res (2012) 22(3):177 - 83. Epub 2012/04/13. doi: 10.1097/CMR.0b013e328352dbef. PubMed PMID: 22495668.

12. Puyana C, Denyer S, Burch T, Bhimani AD, McGuire LS, Patel AS, et al Primary Malignant Melanoma of the Brain: A Population-Based Study. World Neurosurg (2019) 130:e1091-e7. Epub 2019/07/20. doi: 10.1016/j.wneu.2019.07.095. PubMed PMID: 31323401.

13. Yamane K, Shima T, Okada Y, Nishida M, Okita S, Hatayama T, et al. Primary pineal melanoma with long-term survival: case report. Surg Neurol. 1994;42(5):433-7. doi:10.1016/0090-3019(94)90353-0. PubMed PMID: 7974151. Epub 1994/11/01.

14. Byun J, Park ES, Hong SH, Cho YH, Kim YH, Kim CJ, et al Clinical outcomes of primary intracranial malignant melanoma and metastatic intracranial malignant melanoma. Clin Neurol Neurosurg (2018) 164:32 - 8. Epub 2017/11/21. doi: 10.1016/j.clineuro.2017.11.012. PubMed PMID: 29156329.

15. Kocher M, Soffietti R, Abacioglu U, Villa S, Fauchon F, Baumert BG, et al. Adjuvant whole-brain radiotherapy versus observation after radiosurgery or surgical resection of one to three cerebral metastases: results of the EORTC 22952-26001 study. J Clin Oncol. 2011;29(2):134-41. doi: 10.1200/JC0.2010.30.1655. PubMed PMID: 21041710; PubMed Central PMCID: PMCPMC3058272.

16. Tang K, Kong X, Mao G, Qiu M, Zhu H, Zhou L, et al. Primary cerebral malignant melanoma: A case report with literature review. Med (Baltim). 2017;96(4):e5805. doi:10.1097/MD.0000000000005805. PubMed PMID: 28121927; PubMed Central PMCID: PMCPMC5287951. Epub 2017/01/26.

17. Troya-Castilla M, Rocha-Romero S, Chocron-Gonzalez Y, Marquez-Rivas FJ. Primary cerebral malignant melanoma in insular region with extracranial metastasis: case report and review literature. World J Surg Oncol. 2016;14(1):235. doi:10.1186/s12957-016-0965-7. PubMed PMID: 27586680; PubMed Central PMCID: PMCPMC5009555. Epub 2016/09/03.

18. Di Giacomo AM, Ascierto PA, Pilla L, Santinami M, Ferrucci PF, Giannarelli D, et al. Ipilimumab and fotemustine in patients with advanced melanoma (NIBIT-M1): an open-label, single-arm phase 2 trial. Lancet Oncol. 2012;13(9):879-86. Epub 2012/08/17.. ) ). -2045(12)70324-8. PubMed PMID: 22894884. doi: 10.1016/S1470. 
19. Guirguis LM, Yang JC, White DE, Steinberg SM, Liewehr DJ, Rosenberg SA, et al Safety and efficacy of high-dose interleukin-2 therapy in patients with brain metastases. J Immunother(2002) 25(1):82 7. Epub 2002/04/02. doi: 10.1097/00002371-200201000-00009. PubMed PMID: 11924913; PubMed Central PMCID: PMCPMC2424228.

20. Hodi FS, O'Day SJ, McDermott DF, Weber RW, Sosman JA, Haanen JB, et al Improved survival with ipilimumab in patients with metastatic melanoma. N Engl J Med (2010) 363(8):711 - 23. Epub 2010/06/08. doi: 10.1056/NEJMoa1003466. PubMed PMID: 20525992; PubMed Central PMCID: PMCPMC3549297.

21. Hong JJ, Rosenberg SA, Dudley ME, Yang JC, White DE, Butman JA, et al. Successful treatment of melanoma brain metastases with adoptive cell therapy. Clin Cancer Res. 2010;16(19):4892-8. doi:10.1158/1078-0432.CCR-10-1507. PubMed PMID: 20719934; PubMed Central PMCID: PMCPMC6291850. Epub 2010/08/20.

22. Robert C, Thomas L, Bondarenko I, O'Day S, Weber J, Garbe C, et al. Ipilimumab plus dacarbazine for previously untreated metastatic melanoma. N Engl J Med. 2011;364(26):2517-26. doi:10.1056/NEJMoa1104621. PubMed PMID: 21639810. Epub 2011/06/07.

23. Chapman PB, Hauschild A, Robert C, Haanen JB, Ascierto P, Larkin J, et al. Improved survival with vemurafenib in melanoma with BRAF V600E mutation. N Engl J Med. 2011;364(26):2507-16. doi:10.1056/NEJMoa1103782. PubMed PMID: 21639808; PubMed Central PMCID: PMCPMC3549296. Epub 2011/06/07.

24. Kirkwood JM, Bastholt L, Robert C, Sosman J, Larkin J, Hersey P, et al. Phase II, open-label, randomized trial of the MEK1/2 inhibitor selumetinib as monotherapy versus temozolomide in patients with advanced melanoma. Clin Cancer Res (2012) 18(2):555-67. Epub 2011/11/04. doi:10.1158/1078-0432.CCR-11-1491. PubMed PMID: 22048237; PubMed Central PMCID: PMCPMC3549298.

25. Rulli E, Legramandi L, Salvati L, Mandala M. The impact of targeted therapies and immunotherapy in melanoma brain metastases: A systematic review and meta-analysis. Cancer. 2019;125(21):377689. doi:10.1002/cncr.32375. PubMed PMID: 31287564. Epub 2019/07/10.

\section{Figures}



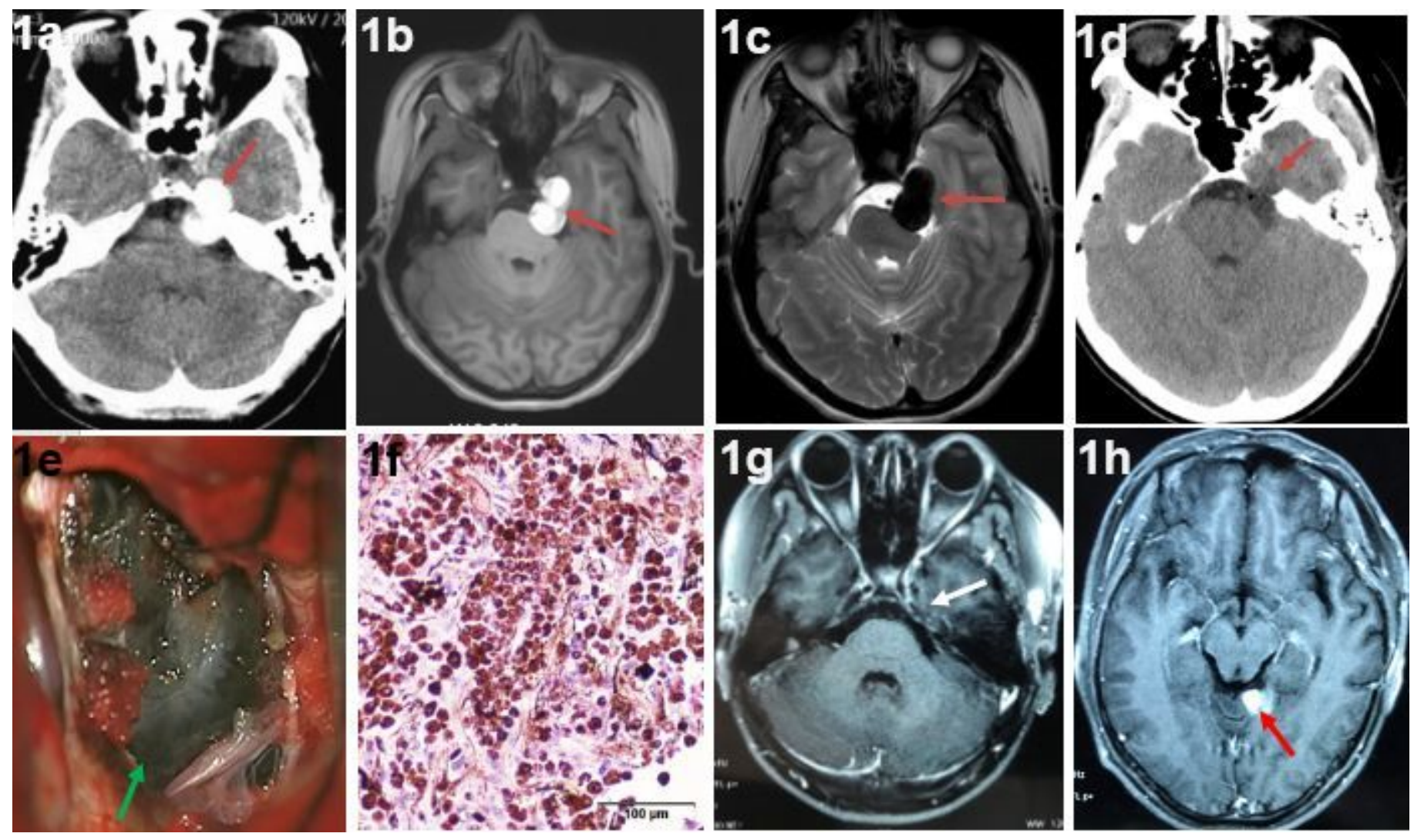

\section{Figure 1}

Preoperative, intraoperative and postoperative imaging and pathological examination of a 29-year-old female patient with left petrous apex melanoma. a) Preoperative axial CT showed a high-density lesion (red arrow). b) Preoperative axial T1WI showed an irregular high signal lobulated lesion (red arrow). c) Preoperative axial T2WI showed an irregular low signal lobulated lesion (red arrow). d) Axial CT at 1 day after surgery showed total tumor resection (red arrow). e) Intraoperative imaging showed melanoma (green arrow) with clear boundary. f) Postoperative Hematoxylin and eosin (H\&E) staining at $\times 100$ showed the tumor tissue rich in melanin. g) Axial T1-weighted contrast-enhanced MRI at 12 months after surgery showed total tumor resection (white arrow). h) Axial T1-weighted contrast-enhanced MRI at 22 months after surgery showed melanoma metastasis (red arrow) of tentorial. 

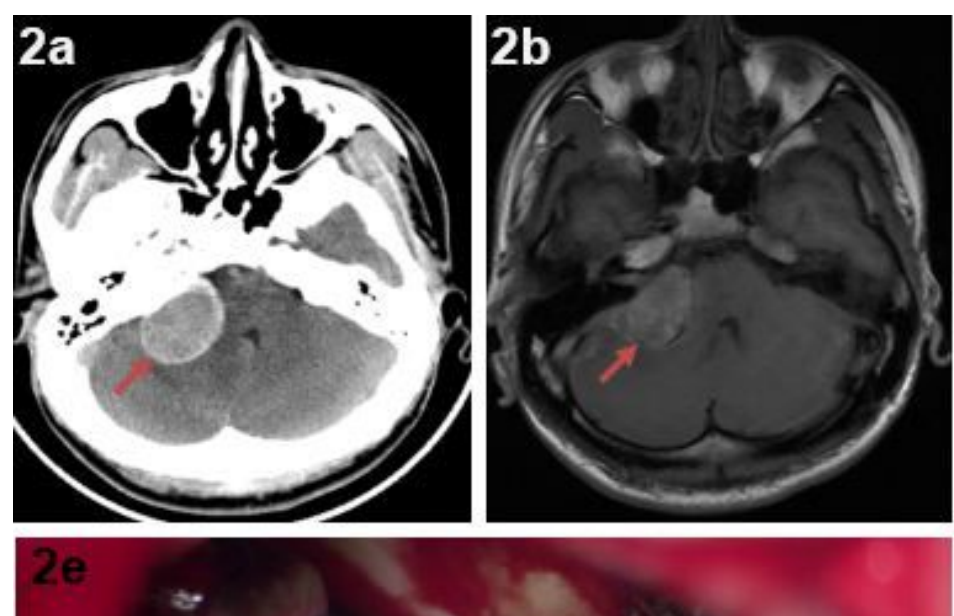

s.
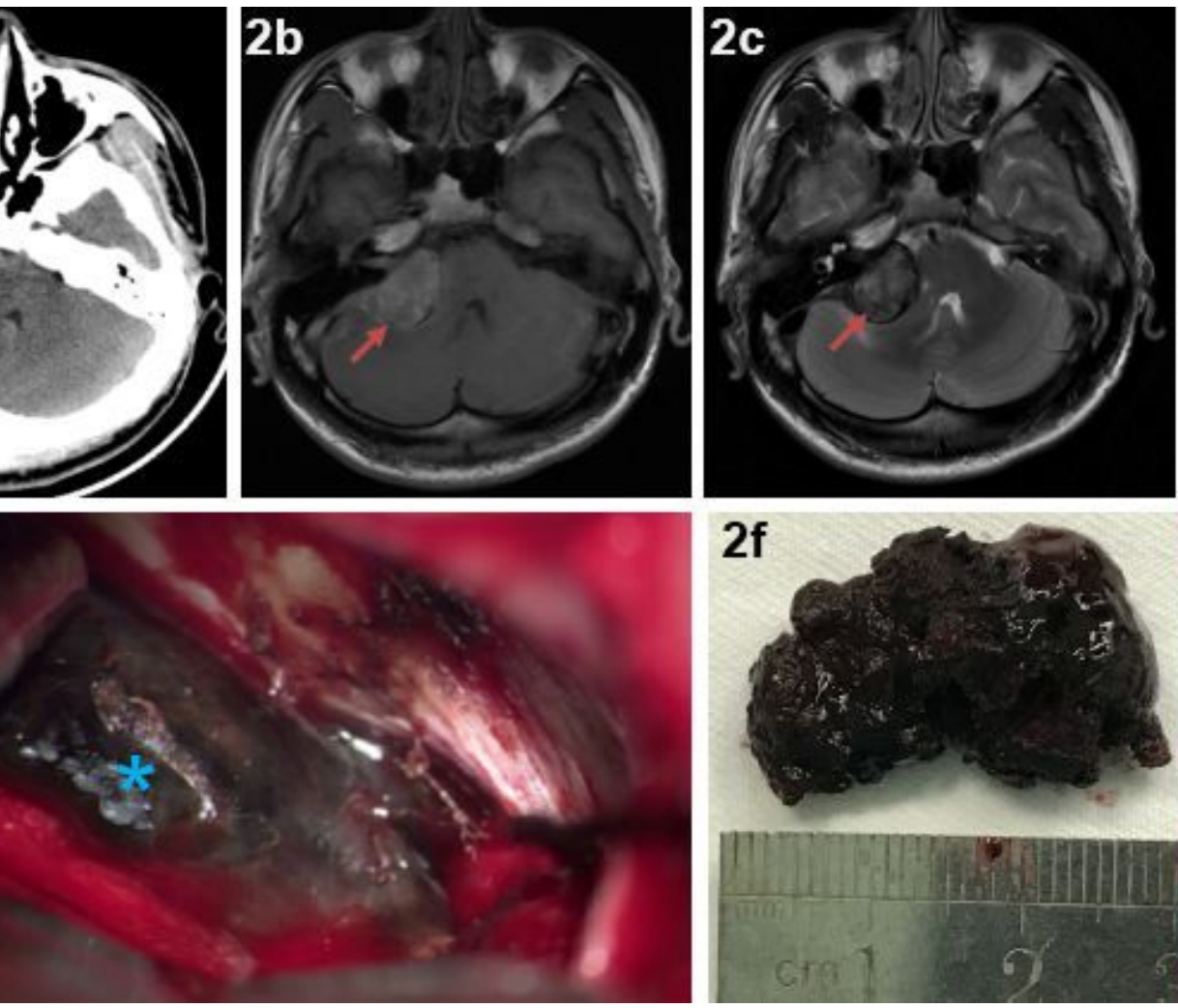

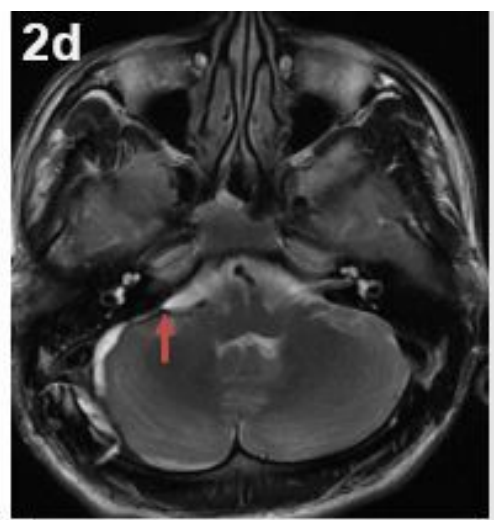

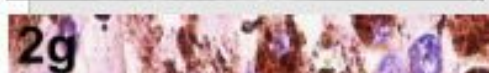

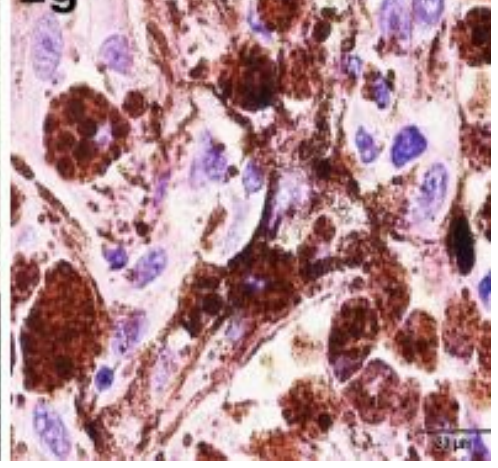

Figure 2

Preoperative and postoperative imaging, specimens and pathological examination of a 28-year-old male patient with right cerebellopontine angle melanoma. a) Preoperative axial CT showed a high-density round lesion (red arrow). b) Preoperative axial T1WI showed a high signal round lesion (red arrow). c) Preoperative axial T2WI showed a low signal round lesion (red arrow). d) Postoperative axial T2WI showed total tumor resection (red arrow). e) Intraoperative imaging showed black melanoma (asterisk). f) Melanoma specimen. g) Postoperative Hematoxylin and eosin (H\&E) staining at $\times 400$ showed heteromorphous large cells with obvious nucleoli. 

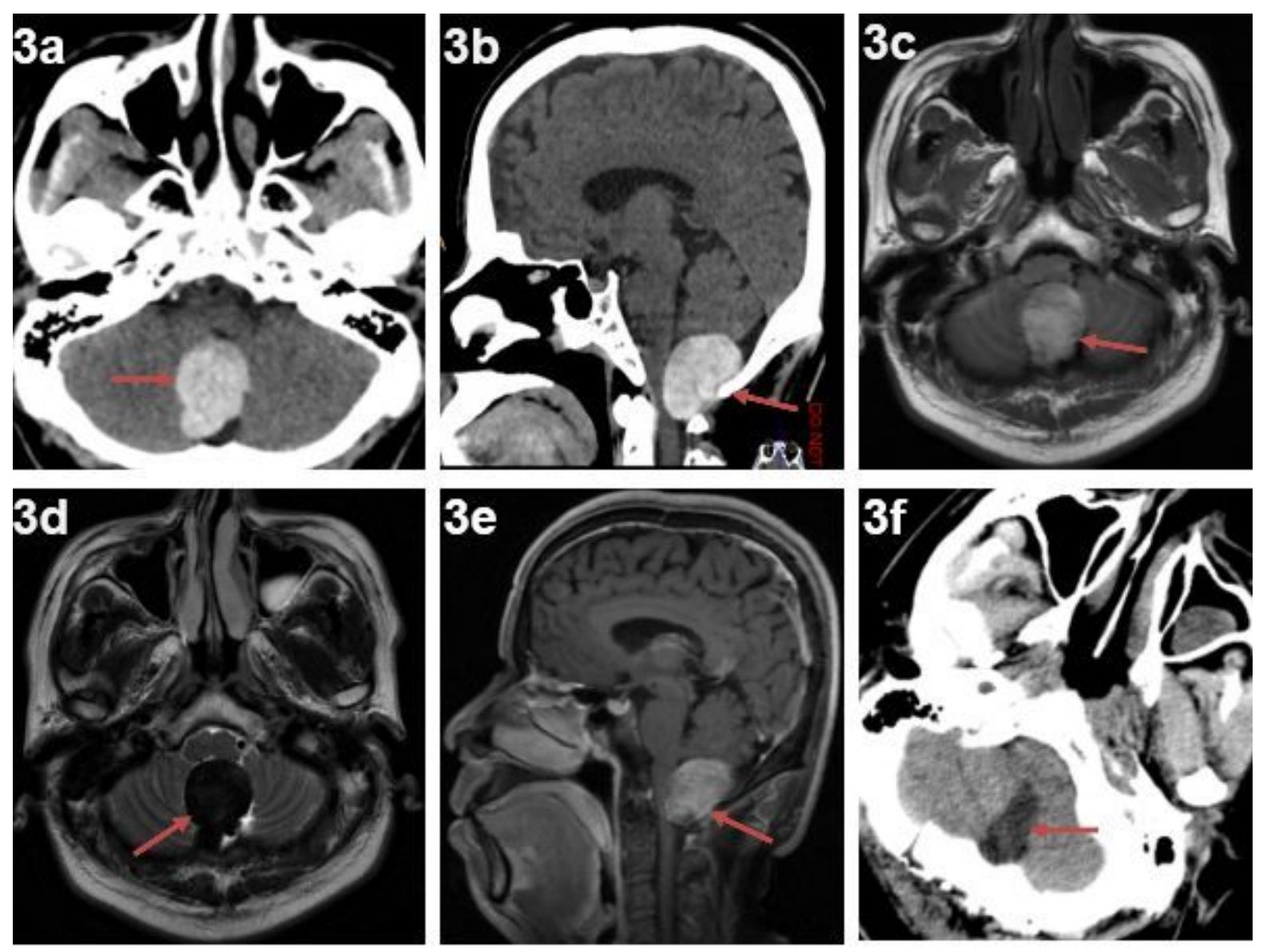

Figure 3

Preoperative and postoperative imaging of a 43-year-old male patient with foramen magnum melanoma. a) Preoperative axial CT showed a high-density round lesion (red arrow). b) Preoperative sagittal CT showed a high-density round lesion (red arrow). c) Preoperative axial T1WI showed a high signal round lesion (red arrow).d) Preoperative axial T2WI showed a low signal round lesion (red arrow). e) Preoperative sagittal T1-weighted contrast-enhanced MRI showed mild enhancement lesion (red arrow). f) Axial CT at 1 day after surgery showed total tumor resection (red arrow). 

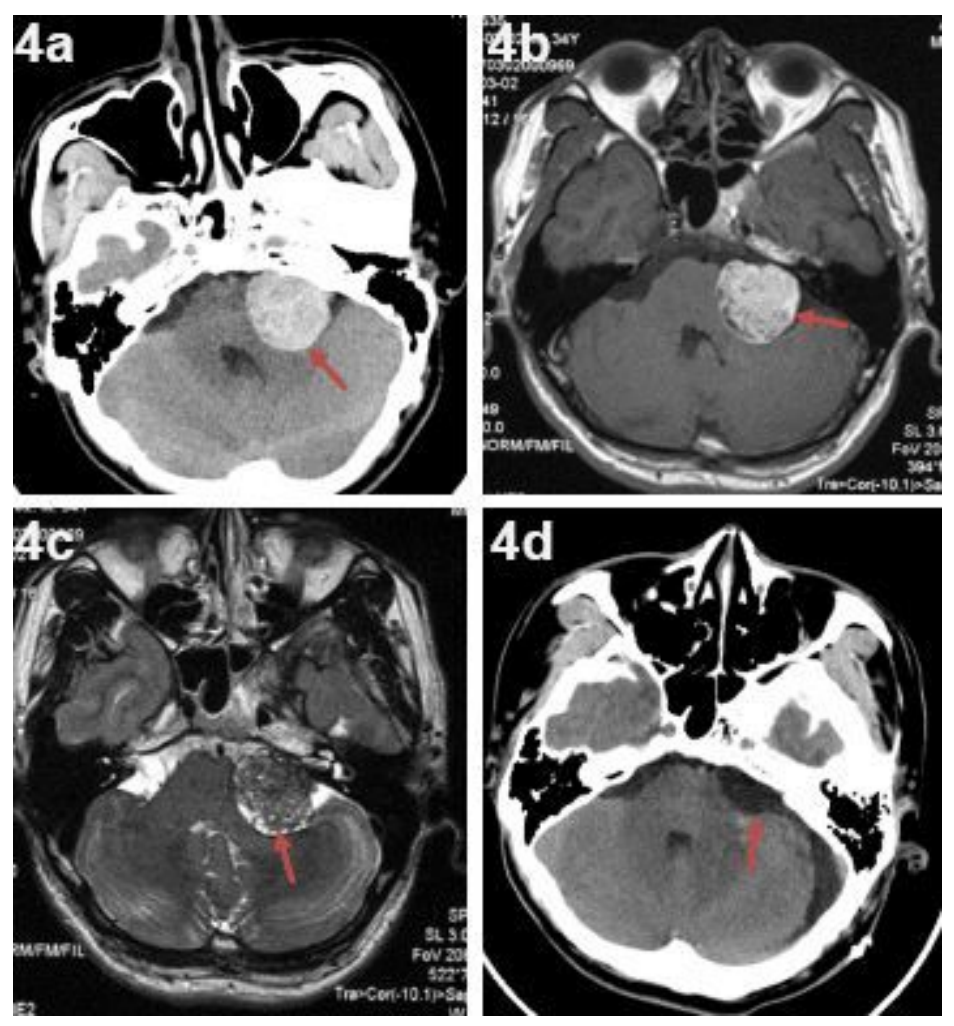

Figure 4

Preoperative and postoperative imaging of a 34-year-old male patient with left cerebellopontine angle melanoma. a) Preoperative axial CT showed a high-density round lesion (red arrow). b) Preoperative axial T1WI showed a high signal round lesion (red arrow).c) Preoperative axial T2WI showed a low signal round lesion (red arrow). d) Axial CT at 1 day after surgery showed total tumor resection (red arrow). 


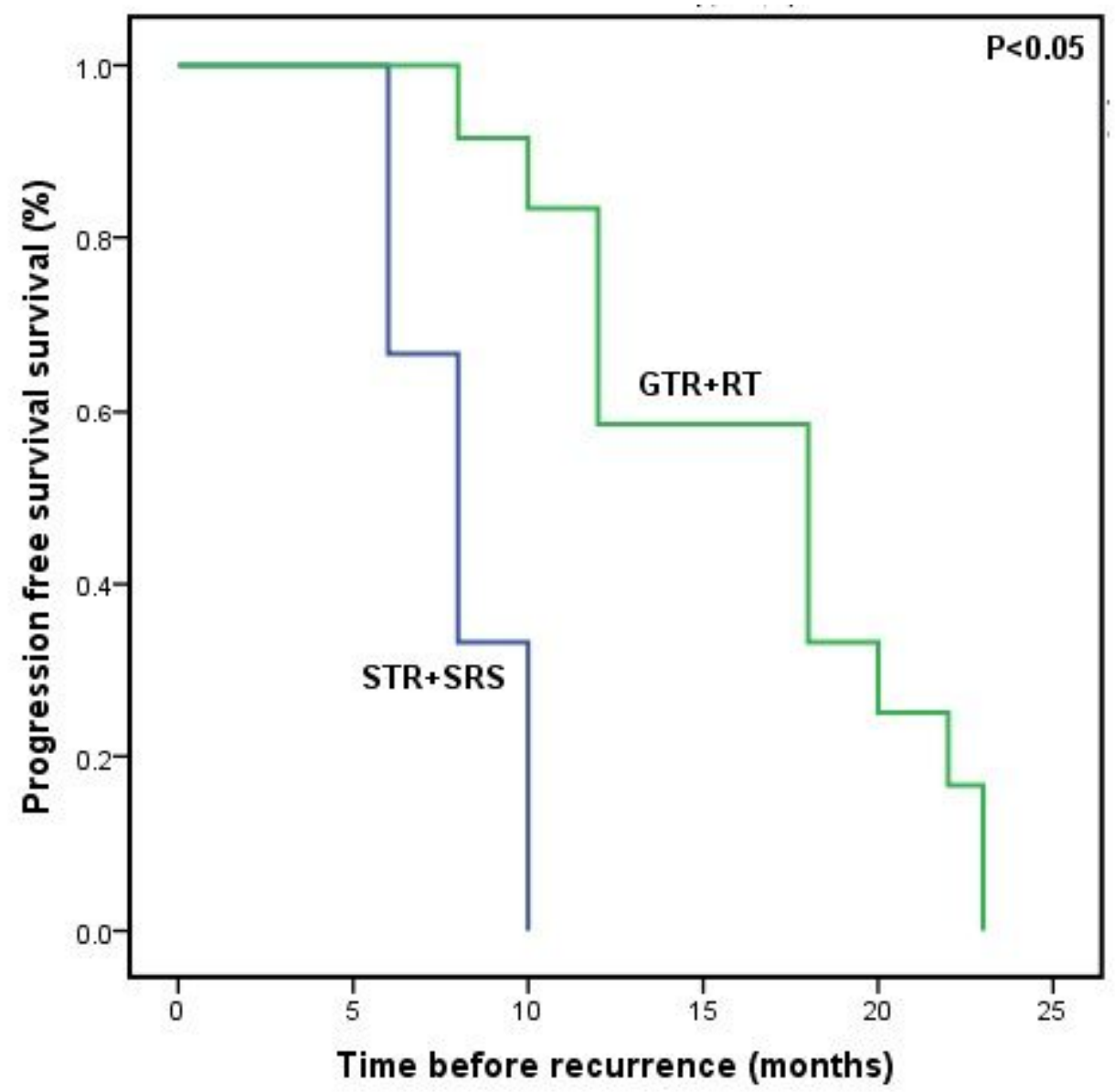

Figure 5

The Kaplan-Meier curves of extended progression free survival of patients were treated with gross total resection (GTR) with radiotherapy (RT). 


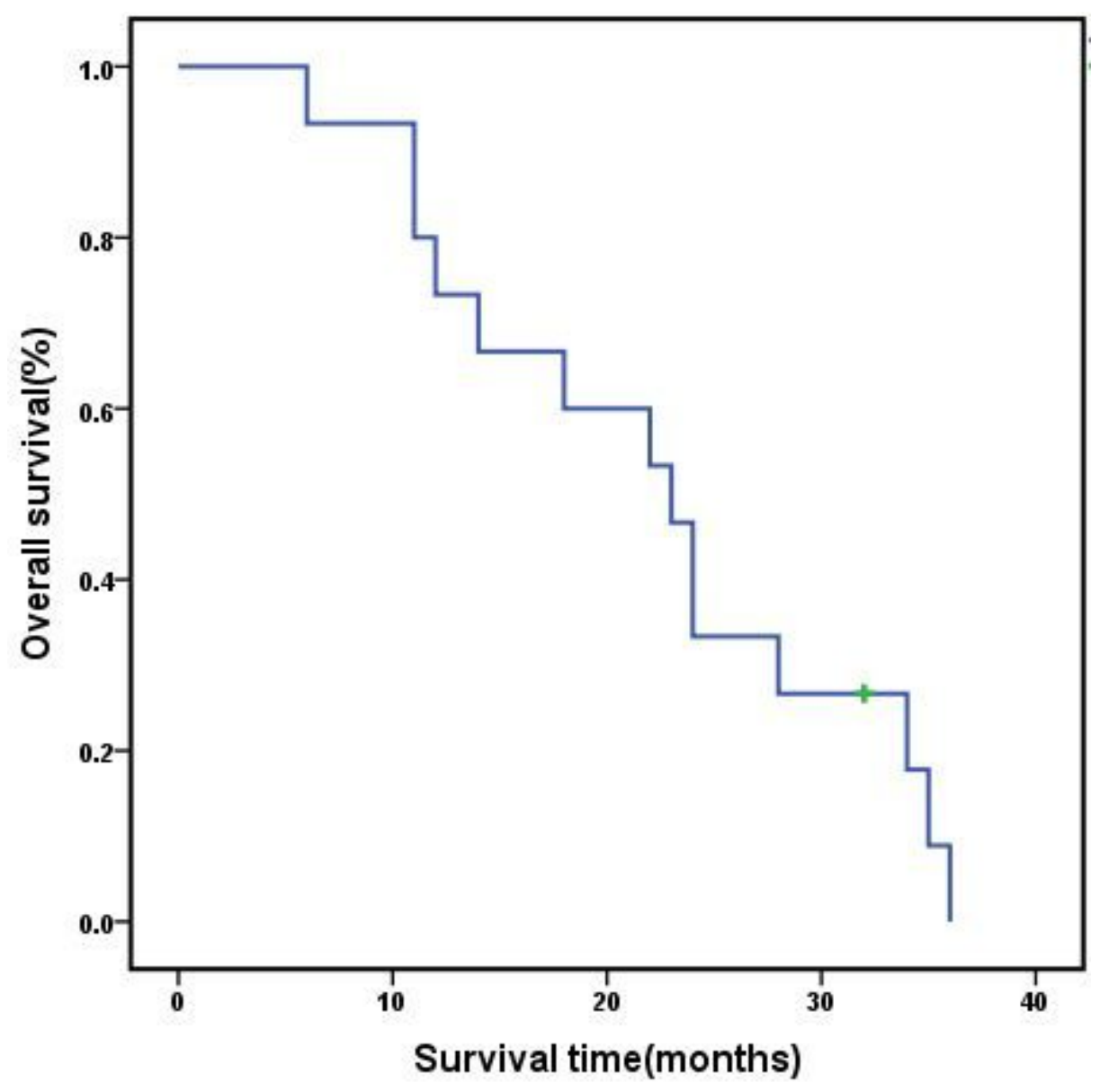

Figure 6

The Kaplan-Meier curve of overall survival of the 15 patients. 


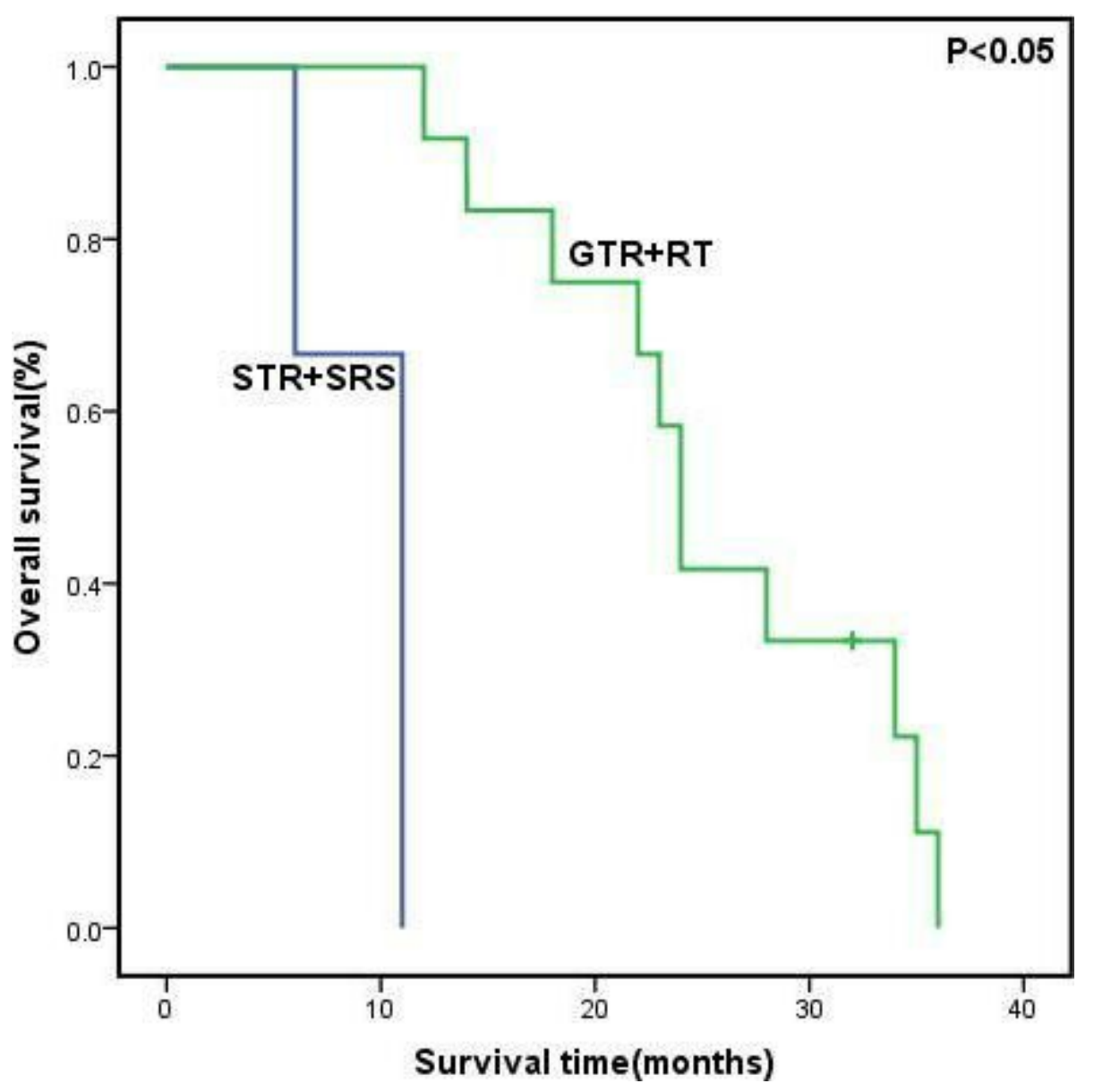

Figure 7

The Kaplan-Meier curves of extended overall survival of patients were treated with gross total resection (GTR) with radiotherapy (RT). 